Orthopäde 2008 · 37:525-531

DOI 10.1007/s00132-008-1235-3

Online publiziert: 1 . Juni 2008

c) Springer Medizin Verlag 2008

P. Henle $\cdot$ M. Tannast $\cdot$ K.A. Siebenrock

Klinik für Orthopädische Chirurgie, Inselspital, Universitätsspital Bern, Schweiz

\title{
Bildgebende Diagnostik der Hüftdysplasie
}

males Hüftgelenk - Pincer-Impingement zulassen.

\section{Konventionelle \\ Röntgenbildgebung}

\section{Beckenübersicht anterior- posterior (a.-p.)}

Die wichtigste Projektion zur Erkennung von Pathologien des Hüftgelenks ist die Beckenübersicht. Hierfür liegt der Patient in Rückenlage und die Hüftgelenke befinden sich in einer Innenrotationsstellung von $15^{\circ}$ zur Kompensation der femoralen Antetorsion. Der Zentralstrahl wird bei einem Film-Fokus-Abstand von 1,2 m auf den Mittelpunkt einer gedachten Linie zwischen der Verbindung beider Spinae iliacae superiores sowie der Symphyse gerichtet. Kriterien für eine qualitativ akzeptable Aufnahmetechnik sind die symmetrische Abbildung der Foramina obturatoria, der Beckenkämme sowie eine rotationsfreie Darstellung beider Schenkelhälse. Die Spitze des Os coccygeum sollte sich auf der Mittellinie über der Symphyse in einem Abstand von 1-2 cm befinden, um eine Fehlinterpretation durch eine übermäßige Beckenkippung zu verhindern [15].

Wichtige Messparameter zur Beurteilung der femoralen Überdachung auf dieser Projektion sind (• Tab. 1):

- der CE-Winkel (LCE-angle) nach Wiberg [21],

- der Tragflächenwinkel (TF) oder azetabulärer Index (AI) [19],

- der Tiefe-zu-Weite-Index nach Heyman u. Herndon [8],

- der Femurkopfextrusionsindex nach Heyman u. Herndon [8],

- das „cross-over sign“.

\section{CE-Winkel}

Der Zentrum-Erker-Winkel nach Wiberg wird im englischen Sprachraum als „lateral center-edge angle" (LCE angle) bezeichnet [21]. Er beschreibt den Winkel zwischen einer Linie durch das Hüftkopfzentrum und den Pfannenerker sowie der Vertikalen (• Abb. 1). Wiberg beschrieb Werte $<20^{\circ}$ als Zeichen einer azetabulären Dysplasie, zwischen 20 und $25^{\circ}$ als grenzwertig und $>25^{\circ}$ als normal. Bei Winkeln $>39^{\circ}$ besteht jedoch aufgrund der ausgedehnten Überdachung die Gefahr eines FAI $[18,20]$. phologie auf der Achse Dysplasie - nor-

\section{Tab. 1 Messparameter zur Beurteilung der Hüftkopfüberdachung auf konventionellen Röntgenaufnahmen}

\begin{tabular}{|c|c|c|c|c|c|}
\hline & Parameter & Dysplasie & Norm & FAI & Literatur \\
\hline \multirow[t]{5}{*}{ Beckenübersicht } & CE-Winkel $\left[^{\circ}\right]$ & $<25$ & $25-39$ & $>39$ & {$[18,20,21]$} \\
\hline & Tragflächenwinkel $\left[^{\circ}\right]$ & $>10$ & $0-10$ & $\leq 0$ & {$[9,18]$} \\
\hline & „Al of depth to width“ [\%] & $<40$ & $40-60^{\circ}$ & Nicht validiert & {$[8,13]$} \\
\hline & Femurkopfextrusionsindex [\%] & $>25$ & $10-26$ & $<10$ & {$[8,13,17]$} \\
\hline & Cross-over sign & \pm & - & + (Pincer) & {$[11,12,16]$} \\
\hline Faux-profil & VCA-Winkel $\left[{ }^{\circ}\right]$ & $<25$ & $>25$ & Nicht validiert & {$[9,10]$} \\
\hline \multirow[t]{3}{*}{ Cross-table } & „Linear indentation sign“ & - & - & + (Pincer) & [18] \\
\hline & a-Winkel $\left[{ }^{\circ}\right]$ & & $<50$ & $>50$ (Cam) & [14] \\
\hline & Kopf-Hals-Offset [mm] & & $>10$ & $<10$ (Cam) & [6] \\
\hline
\end{tabular}


Orthopäde 2008 · 37:525-531

DOI 10.1007/s00132-008-1235-3

(c) Springer Medizin Verlag 2008

P. Henle - M. Tannast · K.A. Siebenrock Bildgebende Diagnostik der Hüftdysplasie

Zusammenfassung

Die detaillierte bildgebende Darstellung von Pathomorphologien des Hüftgelenks stellt einen wichtigen diagnostischen Baustein zur Bewertung und therapeutischen Entscheidungsfindung bei Hüftgelenkbeschwerden dar. Sowohl die konventionelle Röntgenbildgebung wie auch die Schnittbildgebung inklusive der Magnetresonanzarthrographie sind wertvolle Werkzeuge zur umfassenden diagnostischen Bewertung der Hüftgelenkmorphologie. Morphologische Abweichungen werden anhand der aktuell im Routinebetrieb zum Einsatz kommenden Techniken insbesondere im Hinblick auf die Ausprägung der Hüftgelenküberdachung im Spannungsfeld zwischen Dysplasie und femoroazetabulärem Impingement dargestellt und objektivierbare Parameter vorgestellt.

\section{Schlüsselwörter}

Dysplasie · Hüfte · Femoroazetabuläres Impingement $\cdot$ Pincer $\cdot$ Cam

\section{Imaging in developmental dysplasia of the hip}

\section{Abstract}

Modern imaging techniques are an invaluable tool for assessing pathomorphological changes of the hip. Thorough diagnostic analysis and therapeutic decision making mainly rely on correct interpretation of conventional radiographic projections as well as more modern techniques, including magnetic resonance arthrography. This article gives an overview of the imaging techniques that are routinely used for assessing pathological conditions of the hip, with a special focus on diagnostic findings in developmental dysplasia of the hip as well as in femoroacetabular impingement.

\section{Keywords}

Dysplasia $\cdot$ Hip $\cdot$ Femoroacetabular impingement $\cdot$ Pincer $\cdot$ Cam

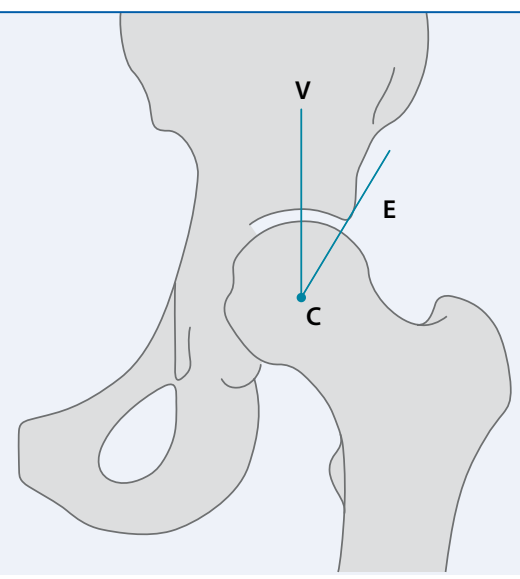

Abb. $1 \Delta$ Der laterale CE-Winkel wird gebildet zwischen einer Linie, die das Hüftkopfzentrum $(C)$ mit dem Pfannenerker $(E)$ verbindet und der Vertikalen $(V)$

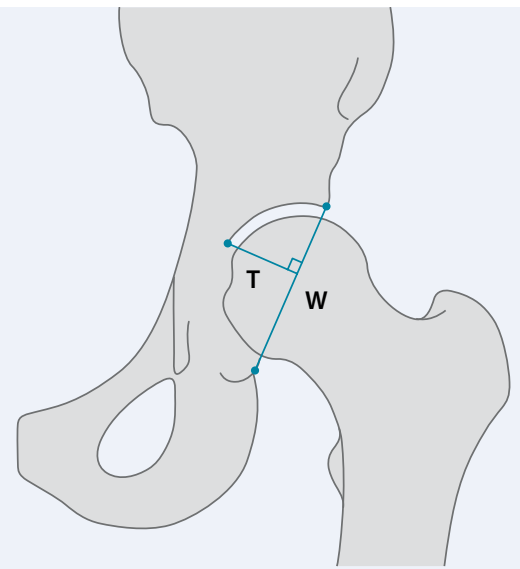

Abb. $3 \Delta$ Der Tiefe-zu-Weite-Index ergibt sich aus dem Quotienten T/W×100

\section{Tragflächenwinkel oder azetabulärer Index}

Als Tragflächenwinkel (TF) nach Tschauner oder Bombelli wird der Winkel zwischen einer Geraden durch den am weitesten medial liegenden Punkt des gewichtstragenden Acetabulums und den Pfannenerker sowie der Horizontalen bezeichnet (- Abb. 2). Der gewichtstragende Teil des Acetabulums ist als Sklerosezone im Bereich des Pfannendaches auf der Beckenübersicht erkennbar. Die Horizontale ist durch eine beide Hüftkopfzentren verbindende Linie oder durch die Tangente an beide Tränenfiguren gegeben. Der Tragflächenwinkel wird auch als ,horizontal toit externe" (HTE) nach Lequesne [9] oder als ,acetabular index (AI) of the weightbearing surface" (nicht zu verwechseln mit dem ,acetabular index

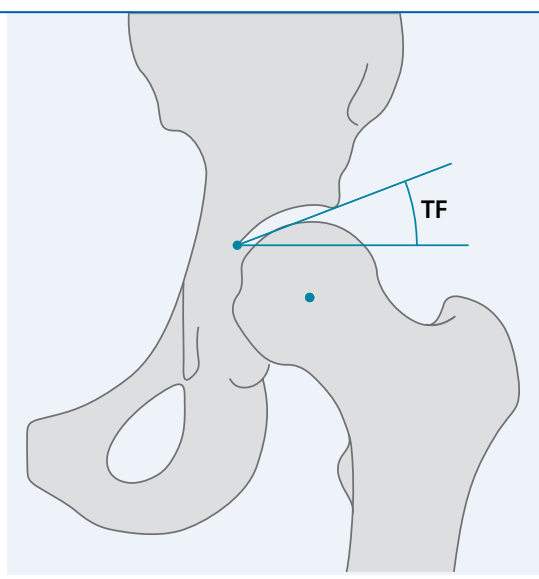

Abb. $2 \Delta$ Der TF oder Al wird zwischen einer Parallelen zum gewichtstragenden Anteil des Acetabulums und der Horizontalen gebildet

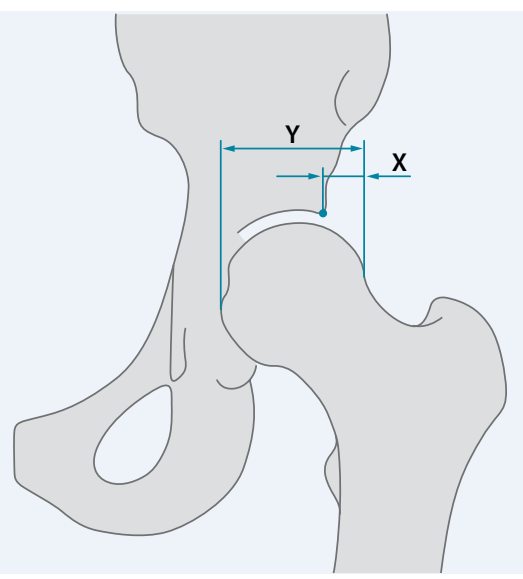

Abb. $4 \Delta$ Der FEl ergibt sich aus dem Quotienten $X / Y \times 100$

of depth to width", s. unten) bezeichnet. Während eine Dysplasie durch Winkel $>10^{\circ}$ gekennzeichnet ist, muss bei $0^{\circ}$ oder sogar negativen Werten von einer Disposition zum FAI ausgegangen werden.

\section{Tiefe-zu-Weite-Index (,,acetabular index of depth to width")}

Der „acetabular index of depth to width“ wurde erstmals 1950 von Heyman u. Herndon [8] zur Beschreibung der azetabulären Morphologie bei Hüften nach einem Morbus Perthes beschrieben und von Murphy et al. [13] zur Beschreibung dysplastischer Hüften angepasst. Er errechnet sich aus dem Quotienten von Tiefe/Durchmesser des Acetabulums multipliziert mit 100 (ब Abb. 3). Murphy et al. [13] konnten zeigen, dass bei Patienten, die im Alter von 65 Jahren eine schwe- 
re Koxarthrose entwickelt hatten, der Index $31 \pm 7 \%$ (19-53\%) im nicht betroffenen Kontrollkollektiv dagegen $48 \pm 6 \%$ (38$62 \%$ ) betrug.

\section{Femurkopfextrusionsindex}

Der Femurkopfextrusionsindex (FEI, „femoral head extrusion index") wurde ebenfalls von Heyman u. Herndon [8] zur Beschreibung von Hüften nach Morbus Perthes verwendet. Er beschreibt den nicht überdachten Anteil des Hüftkopfes in Prozent im Verhältnis zum gesamten Kopfdurchmesser (• Abb. 4). Normale Hüftgelenke zeigen einen nicht überdachten Anteil von $<25 \%$. Im bereits erwähnten Kollektiv von Murphy et al. [13] wurde bei Patienten mit Dysplasiekoxarthrose ein FEI von $36 \pm 12 \%$ (15-62\%), in der Kontrollgruppe von $12 \pm 8 \%(\mathrm{o}-31 \%)$ ermittelt. Bei einem FEI von $<10 \%$ besteht das Risiko einer zu großen Überdachung, was wiederum zum FAI prädisponiert [17].

\section{"Cross-over sign"}

Durch die genauere Betrachtung oder Markierung des vorderen und hinteren Pfannenrandes können unterschiedliche Ausrichtungen (Versionen) des Acetabulums voneinander unterschieden werden. Im Normalfall projiziert sich durch die Anteversion des Acetabulums der Pfannenvorderrand medial des hinteren Pfannenrandes. Besteht eine zu ausladende anterosuperiore Überdachung, liegt die vordere Pfannenrandlinie kranial zunächst lateral des hinteren Pfannenrandes, um diese im Verlauf nach kaudal zu überkreuzen. Diese Überkreuzung von vorderer und hinterer Pfannenrandprojektion als Zeichen einer kranialen, azetabulären Retroversion wird als „cross-over sign“ bezeichnet (• Abb.5). Eine Retroversion des Acetabulums kann auch in Kombination mit einer Pfannendysplasie vorliegen $[11,12,16]$.

\section{Faux-profil-Aufnahme}

Ergibt sich in der Beckenübersicht der Verdacht auf ein laterales Überdachungsdefizit, empfiehlt sich die Anfertigung einer Faux-profil- (False-profile-)Aufnahme der betroffenen Hüfte [10]. Die Aufnahme wird im Stehen angefertigt, wobei die Platte der abzubildenden Hüfte aufliegt. Patient und Röntgenplatte bilden einen Winkel von $65^{\circ}$, der Fuß wird parallel zur Platte gestellt (Außenrotationsstellung).

Als Kriterium für eine adäquate Aufnahmetechnik wird der Abstand zwischen beiden Hüftkopfzentren verwendet. Dieser sollte dem 2 fachen eines Hüftkopfdurchmessers entsprechen. Im Rahmen der Dysplasiediagnostik lässt sich die anteriore Überdachung mit Hilfe des anterioren CE-Winkels beurteilen [10]. Zur Identifikation eines FAI ist die Faux-profil-Aufnahme bei Vorliegen eines posterioren Impingements durch eine prominente Hinterwand hilfreich. Die im Rah-

\section{Hier steht eine Anzeige.}

\section{盟 Springer}




\section{Leitthema}

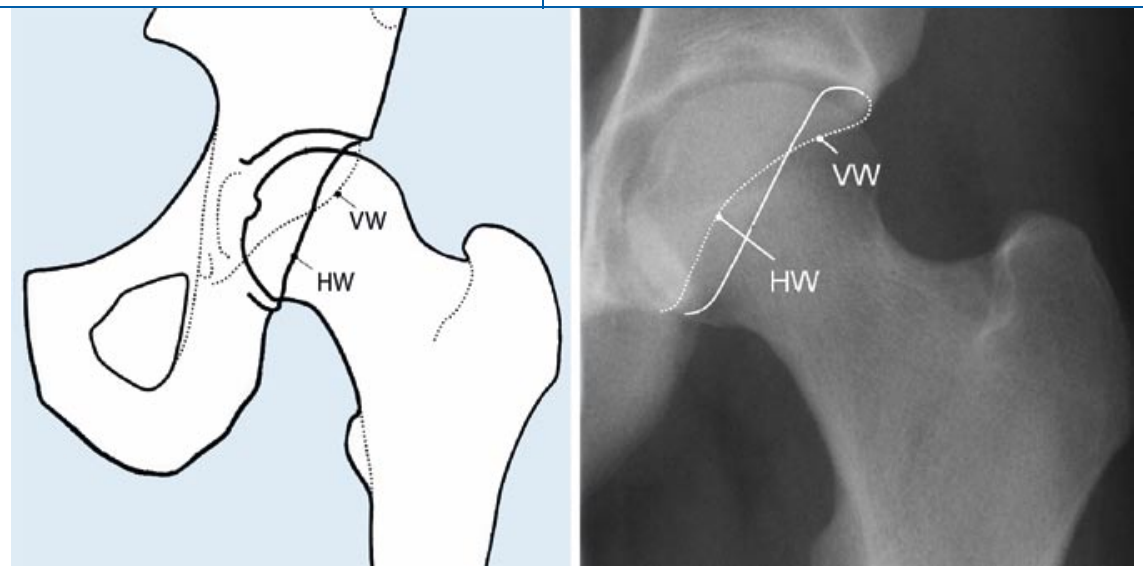

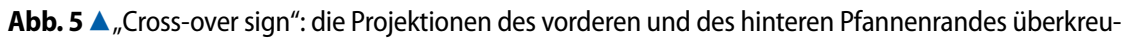
zen sich als Zeichen einer kranialen Retroversion des Acetabulums (VWVorderwand, HW Hinterwand)

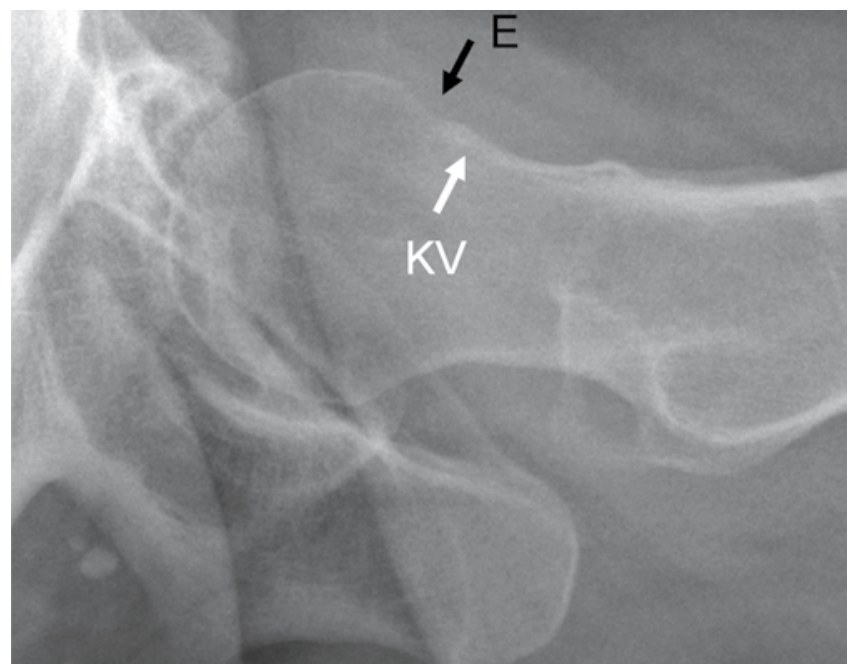

Abb. $7<$ „Linear indentation sign": Gut sichtbar ist die durch das Anstoßen des Kopf-Hals-Übergangs am Acetabulumrand entstandene Einkerbung $(E$, schwarzer Pfeil) sowie die reaktive kortikale Verdickung (KV, weißer Pfeil)

men eines Pincer-Impingements auftretende typische femorale Läsion im dorsokaudalen Anteil des Femurkopfes kann gelegentlich durch eine inferiore Gelenkspaltverschmälerung ebenfalls in dieser Aufnahmetechnik dargestellt werden.

\section{Anteriorer CE-Winkel}

Der anteriore CE-Winkel (VCA-Winkel, verticale-couverture anterieure) nach Lequesne entspricht auf dem lateralen CEWinkel auf der Beckenübersicht [10]. Er wird durch die Linie Hüftkopfzentrum und Pfannenerker mit der Vertikalen gebildet (- Abb. 6). Als Normalwerte gelten Winkel $>25^{\circ}$.

\section{Axiale Projektionen}

Axiale Aufnahmen ergeben zumeist für die Diagnostik einer azetabulären Dysplasie wenig Mehrwert, können jedoch die typischerweise damit einhergehende elliptische Kopfdeformierung darstellen [17]. Zur Beurteilung der femoralen Komponente bzw. zur Bewertung der Asphärizität des Hüftkopfes beim FAI (Cam-Impingement) empfiehlt sich jedoch eine Crosstable-Aufnahme. Hierbei liegt der Patient auf dem Rücken mit dem abzubildenden Bein in Streckstellung und wiederum in $15^{\circ}$ Innenrotation. Das gegenseitige Bein ist in Hüfte und Knie je $90^{\circ}$ gebeugt. Der Zentralstrahl wird horizontal mit einem Winkel von $20^{\circ}$ zur Körperlängsachse sowie einem Film-Fokus-Abstand von 1,2 m auf die Inguinalfalte der zu untersuchenden Hüft gerichtet. Die Platte liegt lateral der Hüfte auf. Zeichen für das Vorliegen eines FAI sind:

- "linear indentation sign“ (Pincer),

- vergrößerter $\alpha$-Winkel, >50 (Cam),

- reduzierter Kopf-Hals-Offset, $<10 \mathrm{~mm}$ (Cam).

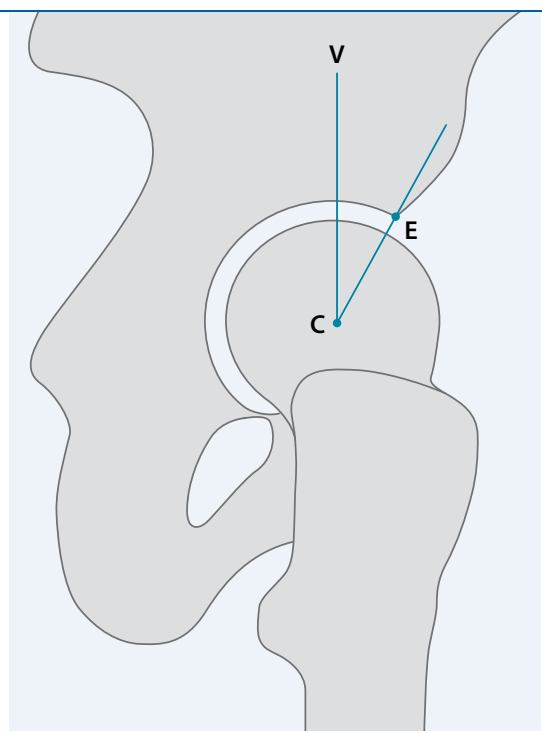

Abb. $6 \triangle$ Der anteriore CE-Winkel wird (analog zum lateralen) gemessen zwischen einer Linie, die das Hüftkopfzentrum $(C)$ mit dem Pfannenerker $(E)$ verbindet und der Vertikalen $(V)$

\section{"Linear indentation sign"}

Das „linear indentation sign“ (indentation = Einkerbung) beschreibt das röntgenmorphologische Korrelat des am Pfannenrand anstoßenden Kopf-Hals-Übergangs. Dabei ist lateral der Einkerbung meist eine Zone reaktiver kortikaler Verdickung sichtbar (• Abb. 7).

\section{a-Winkel}

Der $\alpha$-Winkel wird auf der Cross-tableProjektion zwischen einer Linie durch die Femurhalsachse sowie einer weiteren Linie gebildet, die vom Hüftkopfzentrum durch den Punkt verläuft, an dem die Kopfkontur die Sphärizität verlässt (- Abb. 8). Ergibt sich hierbei ein Winkel von $>50^{\circ}$, besteht die Gefahr, dass sich in Innenrotation und Flexion der asphärische Kopfanteil in die Pfanne eindreht, was einem FAI vom Cam-Typ entspricht [14].

\section{Kopf-Hals-Offset}

Zur Bestimmung des Kopf-Hals-Offsets werden 2 Parallelen zur Schenkelhalsachse gelegt. Einerseits als Tangente an die anteriore Kopfkontur, zum anderen durch den Punkt, an dem die Kopf-Hals-Kontur die Sphärizität des Femurkopfes verlässt (vgl. $\alpha$-Winkel). Der Abstand zwischen den beiden Parallelen entspricht dem Kopf-Hals-Offset (• Abb. 8). Im 
Abb. 8 Bestimmung von a-Winkel und Kopf-HalsOffset auf axialen Projektionen (cross table): a mit Normalbefund, b mit reduziertem Offset und vergrößertem a-Winkel
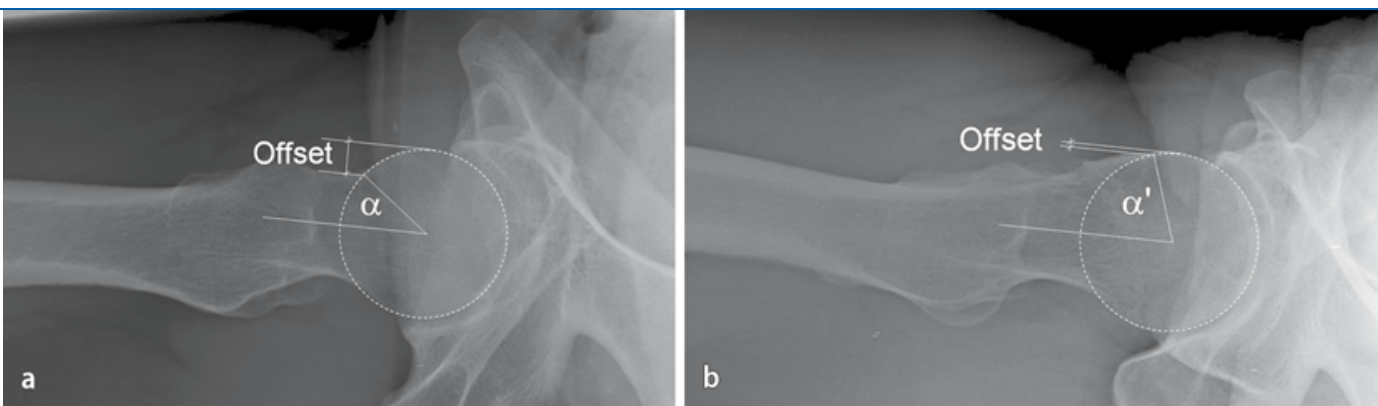

Normalkollektiv wurden für diesen Parameter Werte von $11,6 \pm 0,7 \mathrm{~mm}$ bestimmt, bei Vorliegen eines Cam-Impingements reduziert sich der Kopf-Hals-Offset auf 7,2 $\pm 0,7 \mathrm{~mm}$ [6]. Als klinische Faustregel gilt: Ein Kopf-Hals-Offset von $<10 \mathrm{~mm}$ gilt als Indikator für das Vorliegen eines FAI.

\section{Computertomographie}

Die Computertomographie (CT) kann weitere Hinweise auf fokale Überdachungsdefizite geben. Aufgrund der mit Ihr verbundenen Strahlenbelastung bedarf ihr Einsatz jedoch - insbesondere in einem jungen Patientenkollektiv - einer besonderen Rechtfertigung, die eher selten gegeben sein dürfte. Für die dreidimensionale Planung von Korrektureingriffen am Becken kann die CT jedoch in der Zukunft durchaus eine wertvolle Hilfe darstellen.

\section{AASA, PASA und HASA}

Anda et al. $[2,3,4]$ führten ein Bewertungssystem zur Erfassung einer azetabulären Dysplasie auf CT-Schnittbildern ein. Die Bestimmung der entsprechenden Winkel erfolgt auf einer durch beide Hüftkopfzentren gelegten axialen Schicht. Vom jeweiligen Hüftkopfzentrum aus wird je eine Tangente an den vorderen und hinteren Pfannenrand gelegt. Die Gerade zum vorderen Pfannenrand bildet mit der Verbindungslinie zwischen beiden Hüftköpfen den AASA (anterior acetabular sector angle), entsprechend die Gerade zum hinteren Pfannenrand den PASA (posterior acetabular sector angle). Die Summe aus beiden Winkeln wird als HASA (horizontal acetabular sector angle) bezeichnet ( $\bullet$ Abb. 9).

Abb. 9 Quantifizierung der azetabulären Überdachung auf CTSchnittbildern: Der AASA bestimmt die anteriore, der PASA die posteriore Überdachung. Der HASA, als Summe der beiden Winkel, ist ein globaler Überdachungsmarker (DXT rechts, SIN links, $A 1 / 2$ anteriorer Pfannenrand, $C 1 / 2$ Hüftkopfzentrum, $P 1 / 2$ posteriorer Pfannenrand)

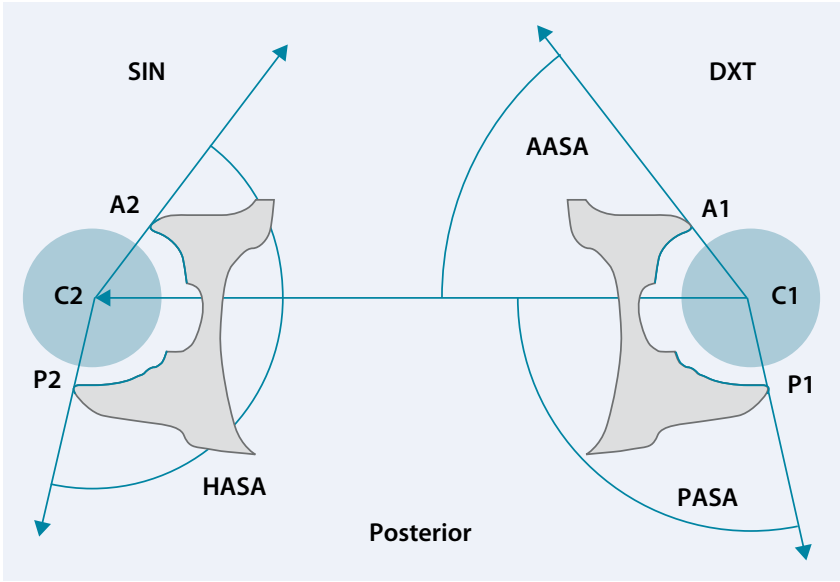

Abb. $10>$ Bestimmung des a-Winkels (schwarze Linien) auf axialen CT-Schnittbildern bei Vorliegen eines ausgeprägten Cam-Impingements

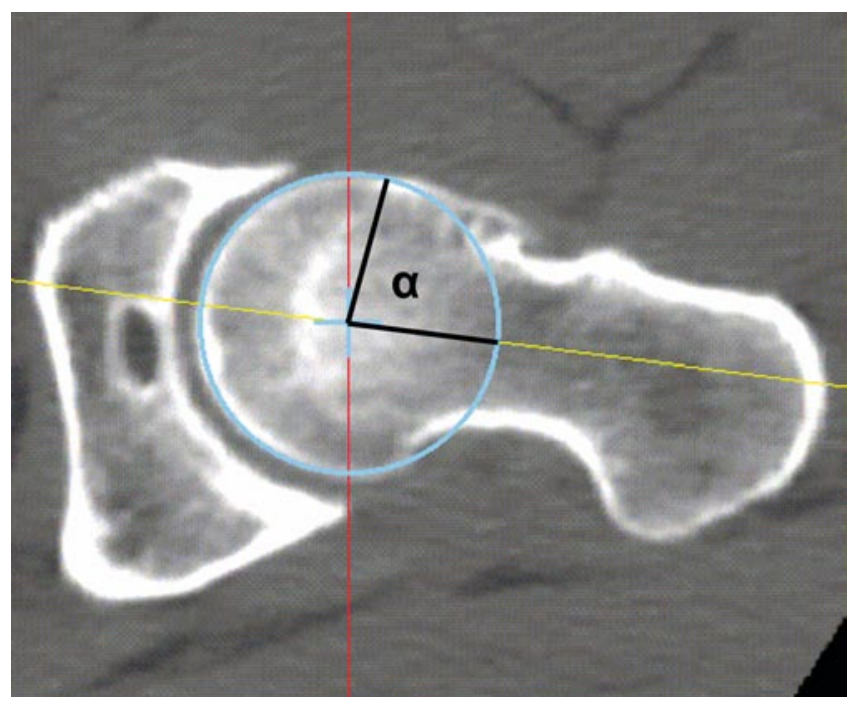

\section{a-Winkel}

Wie auf der Cross-table-Aufnahme in der konventionellen Röntgenbildgebung kann analog auch auf axialen CT-Schichten durch das Hüftkopfzentrum der $\alpha$ Winkel bestimmt werden (• Abb. 10). Wie auch bei der konventionellen Bildgebung sind hierbei Werte $>50^{\circ}$ als prädisponierend für ein Cam-Impingement $\mathrm{zu}$ betrachten [14].

\section{Magnetresonanztomographie}

Für die Diagnostik der Hüftdysplasie stellt die Magnetresonanztomographie (MRT) keine Primär- oder Routinediagnostik dar. Die MRT bietet jedoch gewichtige Vorteile, sodass die Zahl der Untersuchungen in diesem Patientengut im Steigen begriffen ist. So bietet die MRT ohne Einsatz ionisierender Strahlung die Möglichkeit der Abbildung von periartikulären Weichteilstrukturen, was bei der Beurteilung der Knorpelqualität sowie des Labrum ace- 


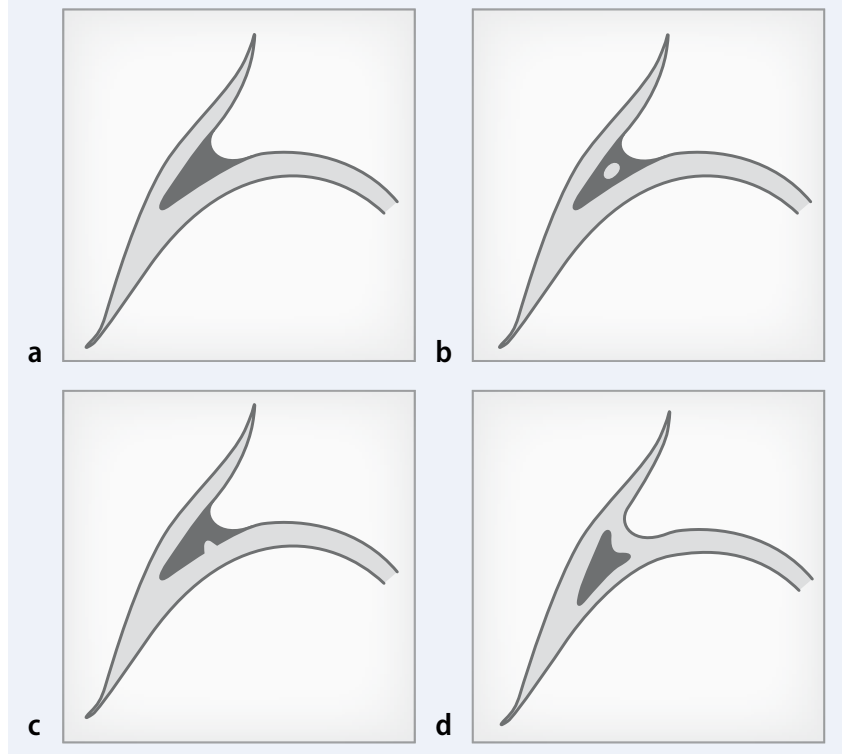

Abb. $11<$ Einteilung der Labrumläsionen im MRA-Bild nach Czerny [5]: a Labrum normal, b Stadium I, c Stadium II, d Stadium III tabulare von höchstem Interesse ist. Dabei ist für die qualitativ hochwertige Diagnostik die Durchführung einer MR-Arthrographie (MRA) unerlässlich.

\section{MR-Arthrographie}

Für die MRA werden unter Durchleuchtungskontrolle 10-20 ml eines Gadolinium-haltigen Kontrastmittels in das zu untersuchende Hüftgelenk injiziert. Die Untersuchung erfolgt dann üblicherweise mit einer Oberflächenspule. Eine radiale Schnittführung erweist sich für die Beurteilung des Labrums ideal. Das normale Labrum zeigt sich in der MRA mit einem dreieckigen Querschnitt und liegt direkt dem knöchernen Rand des Acetabulums an. Zwischen Labrum und Gelenkkapsel ist ein kontrastmittelgefüllter Rezessus erkennbar.

Die MRA ist nach Czerny et al. [5] für die diagnostische Bewertung von Labrumläsionen des Hüftgelenks sensitiver als die native MRI ( $\bullet$ Abb. 11). Die Autoren beschreiben in dieser Studie auch ein Stadienmodell zur Quantifizierung und Qualifizierung von Labrumläsionen. Die Schweregradeinteilung erfolgt anhand der folgenden Einteilung:

- (Stadium o): normales Labrum,

- Stadium I: Signalanhebungen innerhalb des Labrums,

- Stadium II: Labrumeinriss mit KMAnreicherung innerhalb des Labrums,

- Stadium III: kompletter Abriss des Labrums vom Acetabulumrand.

\section{Labrum- und Knorpelläsionen bei azetabulärer Dysplasie}

Bei der Dysplasie besteht insbesondere ventrokranial eine vermehrte Druckund Scherbelastung auf das Labrum acetabulare und den angrenzenden Knorpel. Morphologisches Korrelat dieser Beanspruchung ist eine Verdickung des Labrums mit Einrissen bis hin zur kompletten Ablösung vom knöchernen Acetabulum sowie Läsionen des angrenzend anhaftenden Knorpels („inside out“).

\section{Labrum- und Knorpelläsionen bei FAI}

Labrumläsion sind pathognomonische Veränderungen des FAI - sowohl beim Cam- wie auch beim Pincer-Impingement. Entstehung und Pathomorphologie der Labrumläsionen unterscheiden sich jedoch zwischen den beiden FAI-Typen.

Beim Cam-Impingement entstehen zunächst durch die beim Eindrehen des asphärischen Kopfanteils in das Acetabulum auftretenden Scherkräfte Knorpelläsionen im Bereich des anterosuperioren Pfannenrandes. Durch die sich ausweitende Knorpelavulsion entstehen sekundär Risse oder eine Separation des Knorpels vom weitgehend unveränderten Labrum. Die Knorpelläsionen betreffen hier ein kleineres Segment, reichen jedoch tiefer ins Acetabulum.

Im Gegensatz hierzu ist das Pincer-Impingement eine primäre Pathologie des Labrums. Der am Pfannrand anschlagende Schenkelshals verursacht eine De- genration des Labrums mit Ausbildung von Ganglien innerhalb des Labrums oder einer Ossifikation des Faserknorpels. Knorpelläsionen treten sekundär auch im Rahmen eines Pincer-Impingements auf. Sie sind typischerweise am Femurkopf posteroinferior als sog. „Contrecoup-Läsionen" lokalisiert [7]. Hierbei ist ein größeres Segment betroffen als beim Cam-Impingement, die Veränderungen reichen jedoch weniger in die Tiefe.

\section{Fazit für die Praxis}

Eine adäquate Bildgebung und standardisierte Auswertung des Bildmaterials sind wesentliche Qualitätsmerkmale bei der Differentialdiagnostik von Hüftgelenkbeschwerden. Die technisch einwandfreie Aufnahme ist Grundvoraussetzung für die weitergehende Beurteilung. Dies gilt für alle bildgebenden Verfahren von der konventionellen Projektionsradiographie bis zur MRA. Objektivierbare und evaluierte Messverfahren lassen auf dem so gewonnenen Bildmaterial reproduzierbare diagnostische Bewertungen zu, auf welchen eine optimale Therapieentscheidung aufgebaut werden kann. Der Einsatz der zur Verfügung stehenden Verfahren erfolgt in logisch aufeinander aufbauenden Schritten abhängig von klinischem Untersuchungsergebnis und bereits erhobenen bildgebenden Befunden.

\section{Korrespondenzadresse}

\section{Dr. P. Henle}

Klinik für Orthopädische Chirurgie, Inselspital,

Universitätsspital

Freiburgstraße 3, CH-3010 Bern

Schweiz

philipp.henle@insel.ch

Interessenkonflikt. Der korrespondierende Autor gibt an, dass kein Interessenkonflikt besteht.

\section{Literatur}

1. Anda S (1993) Acetabular dysplasia in the adolescent and young adult. Clin Orthop Relat Res 286: 308-310

2. Anda S, Svenningsen S, Dale LG, Benum P (1986) The acetabular sector angle of the adult hip determined by computed tomography. Acta Radiol Diagn (Stockh) 27(4): 443-447 
3. Anda S, Terjesen T, Kvistad KA (1991) Computed tomography measurements of the acetabulum in adult dysplastic hips: which level is appropriate? Skeletal Radiol 20(4): 267-271

4. Anda S, Terjesen T, Kvistad KA, Svenningsen $S$ (1991) Acetabular angles and femoral anteversion in dysplastic hips in adults: CT investigation. J Comput Assist Tomogr 15(1): 115-120

5. Czerny C, Hofmann S, Neuhold A et al. (1996) Lesions of the acetabular labrum: accuracy of MR imaging and MR arthrography in detection and staging. Radiology 200(1): 225-230

6. Eijer H, Leunig M, Mahomed MN, Ganz K (2001) Crosstable lateral radiograph for screening of anterior femoral head-neck offset in patients with femoro-acetabular impingement. Hip Int 11: 37-41

7. Ganz R, Parvizi J, Beck M et al. (2003) Femoroacetabular impingement: a cause for osteoarthritis of the hip. Clin Orthop Relat Res 417: 112-120

8. Heyman CH, Herndon CH (1950) Legg-Perthes disease; a method for the measurement of the roentgenographic result. J Bone Joint Surg Am 32(4): 767-778

9. Lequesne M (1963) Coxometry measurement of the basic angles of the adult radiographic hip by a combined protractor. Rev Rhum Mal Osteoartic 30: 479-485

10. Lequesne MG, Laredo JD (1998) The faux profil (oblique view) of the hip in the standing position. Contribution to the evaluation of osteoarthritis of the adult hip. Ann Rheum Dis 57(11): 676-681

11. Li PL, Ganz R (2003) Morphologic features of congenital acetabular dysplasia: one in six is retroverted. Clin Orthop Relat Res 416: 245-253

12. Mast JW, Brunner RL, Zebrack J (2004) Recognizing acetabular version in the radiographic presentation of hip dysplasia. Clin Orthop Relat Res 418: 4853

13. Murphy SB, Ganz R, Muller ME (1995) The prognosis in untreated dysplasia of the hip. A study of radiographic factors that predict the outcome. J Bone Joint Surg Am 77(7): 985-989

14. Notzli HP, Wyss TF, Stoecklin CH et al. (2002) The contour of the femoral head-neck junction as a predictor for the risk of anterior impingement. J Bone Joint Surg Br 84(4): 556-560

15. Siebenrock KA, Kalbermatten DF, Ganz R (2003) Effect of pelvic tilt on acetabular retroversion: a study of pelves from cadavers. Clin Orthop Relat Res 407: 241-248

16. Siebenrock KA, Schoeniger R, Ganz R (2003) Anterior femoro-acetabular impingement due to acetabular retroversion. Treatment with periacetabular osteotomy. J Bone Joint Surg Am 85(2): 278-286

17. Steppacher SD, Tannast M, Werlen S, Siebenrock KA (2008) Femoral morphology differs between deficient and excessive acetabular coverage. Clin Orthop Relat Res 466(4): 782-790

18. Tannast M, Siebenrock KA, Anderson SE (2007) Femoroacetabular impingement: radiographic diagnosis--what the radiologist should know. Am J Roentgenol 188(6): 1540-1552

19. Tönnis D (1984) Die angeborene Hüftdysplasie und Hüftluxation in Kindes- und Erwachsenenalter. Springer, Berlin Heidelberg New York

20. Tonnis D, Heinecke A (1999) Acetabular and femoral anteversion: relationship with osteoarthritis of the hip. J Bone Joint Surg Am 81(12): 1747-1770

21. Wiberg G (1939) Studies on dysplastic acetabula and congenital subluxation of the hip joint: With special reference to the complications of osteoarthritis. Acta Chir Scan 58(Suppl): 7-38

\section{Der Unfallchirurg}

Die Zeitschrift „Der Unfallchirurg“ bietet Ihnen jeden Monat umfassende und aktuelle Beiträge zu interessanten Themen aus allen Bereichen der Unfall- und Wiederherstellungschirurgie.

\section{Heft 6/2008:}

- CT-gesteuerte Sakroplastie unter Verwendung insufflierbarer Ballons

- Die Klavikulaschaftfraktur Klassifikation und Therapie

- Visualisierung von Repositionspfaden

- Akzidentelle abdominelle Nadelstichverletzung bei der Entsorgung eines Abwurfcontainers

- Schockraummanagement beim kontaminierten Patienten

- Gewinnung großvolumiger Spongiosamengen zur autologen Knochentransplantation aus dem femoralen Markraum

- CME: Sprunggelenksfrakturen

\section{Heft 7/2008:}

- Der Flexor hallucis longus-Transfer zum Ersatz der Achillessehne

- Die traumatische Schultererstluxation Ergebnisse 6 Monate und 2 Jahre postoperativ nach arthroskopischer Stabilisierung beim jungen Patienten

- Hemipelvektomie - eine Lösung bei Beckenzerreißung

- Knochen im (Über-)Fluss: das seltene Krankheitsbild der Melorheostose

- Tumor oder Trauma. Kasuistik zu synovialen Weichteiltumoren im Kindesalter

- CME: Operative Arthrolyse bei schwergradiger heterotoper Ossifikation nach Hüftgelenksendoprothese

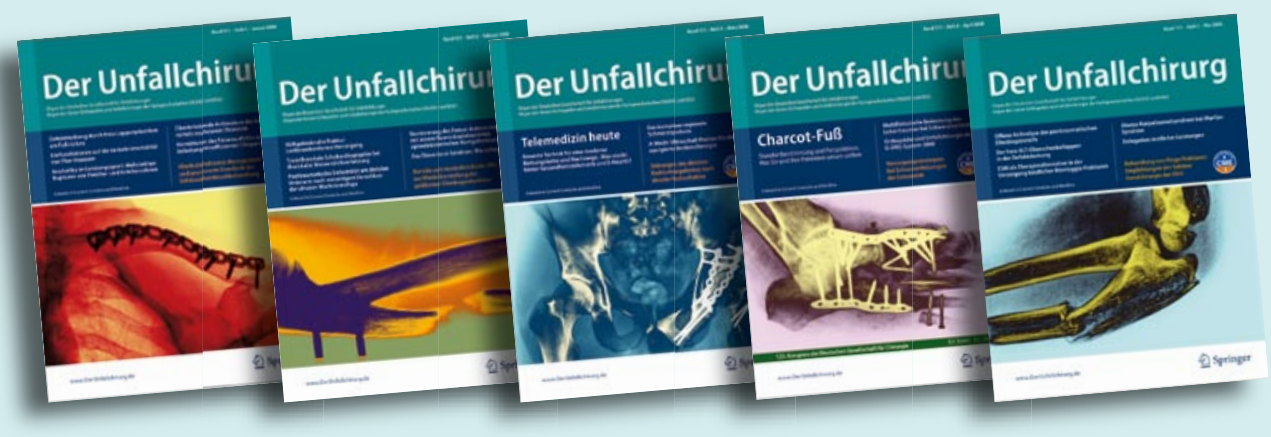

Nutzen Sie unser besonders günstiges Kombinationsabonnement für "Der Orthopäde“ und "Der Unfallchirurg".

Abonnements und Einzelheftbestellung:

\section{Springer Medizin Verlag}

Kundenservice

Haberstr. 7

69126 Heidelberg

Tel. +49/6221/345-4303; Fax: -4229

Subscriptions@springer.com

www.DerUnfallchirurg.de 